\title{
Masa en tránsito en el ventrículo derecho: ¿tumor vs trombo?
}

\author{
Manuel Felipe Cáceres-Acosta ${ }^{1}$ \\ Viviana Quintero-Yepes ${ }^{2}$ \\ Jorge Arroyave-Carvajal ${ }^{2}$ \\ CamiloMadrid-Muñoz ${ }^{3}$ \\ Pedro Abad-Díaz ${ }^{4}$
}

\section{Correspondencia}

Manuel Felipe Cáceres-Acosta, MD

mcaceres55@gmail.com

${ }^{1}$ Fellow de Cardiología Clínica. Universidad CES. Medellín-Colombia.

${ }^{2}$ Especialista en Cardiología Clínica, Universidad CES. Clínica el Rosario. Medellín-Colombia.

${ }^{3}$ Especialista en Cardiología Clínica y Ecocardiografía, Universidad Pontificia Bolivariana. SICOR.

${ }^{4}$ Imagen cardiovascular, Imágenes Diagnósticas SURA, Medellín, Colombia.

Enviado: 19/09/2021.

Aceptado: 10/10/2021

Publicado: 15/12/2021

Citar como: Caceres Acosta M., Quintero Yepes V., Arroyave Carbajal J., Madrid Muñoz C., Abad Diaz P.: Masa en tránsito en el ventrículo derecho: ¿tumor o trombo? RETIC. 2021(Diciembre); 4 (3): 35-38. doi: 10.37615/retic.v4n3a10

Cite this as: Caceres Acosta M., Quintero Yepes V., Arroyave Carbajal J., Madrid Muñoz C., Abad Diaz P.: Right ventricle mass in transit: Tumour or thrombi? RETIC. 2021(December); 4 (3): 35-38. doi: 10.37615/retic.v4n3a10

\section{Palabras clave \\ $\triangleright$ Ventrículo derecho. \\ $\triangleright$ Trombo. \\ $\triangleright$ Neoplasia. \\ $\triangleright$ Diagnóstico.}

\section{RESUMEN}

Las imágenes ecodensas en el VD tienen un amplio espectro de diagnósticos, los principales son tumores o trombos. Se presenta el caso de una mujer de 31 años en la que se diagnostica de forma incidental una masa ecodensa en tránsito en VD sugestiva de trombo. Se ampliaron estudios descartando enfermedad tromboembólica venosa. La resonancia cardíaca confirmó el diagnóstico ecocardiográfico, fue llevada a resección quirúrgica evidenciando trombo antiguo más nuevo y recibió anticoagulación oral indefinida. Este caso muestra como la imagen multimodal permite valorar de forma precisa las masas del VD. El trombo en el VD obliga al clínico descartar enfermedades asociadas.

ABSTRACT
Echodense images in the RV have a wide spectrum of diagnoses, the main ones being tumors or thrombi. We present
the case of a 31-year-old woman in whom an echodense mass in transit in RV suggestive of thrombus was inci-
dentally diagnosed. Venous thromboembolic disease was ruled-out. Cardiac MRI confirmed the echocardiographic
diagnosi. The surgery showed new over old thrombus, and she received indefinite oral anticoagulation. This case
shows how the multimodal imaging allows the accurate assessment of RV masses. The thrombus in the RV forces the
clinician to rule out associated diseases.

\begin{tabular}{|c|l|}
\hline VD & Ventrículo derecho \\
\hline ETT & Ecocardiograma transtorácico \\
\hline RV & Right ventricle \\
\hline TTE & Transthoracic echocardiogram \\
\hline MRI & Magnetic resonance imaging \\
\hline
\end{tabular}

Tabla 1. Cuadro de abreviaturas.

\section{Presentación del caso}

Mujer de 31 años, sin hijos y sin antecedentes conocidos, se encontraba en seguimiento ecocardiográfico por válvula mitral laxa, el cual mostró como hallazgo incidental una masa ecodensa móvil, en tránsito en el VD sugestiva de trombo (ver figura 1, ver videos 1-4), debido al hallazgo se remite a urgencias para ampliación de estudios. 


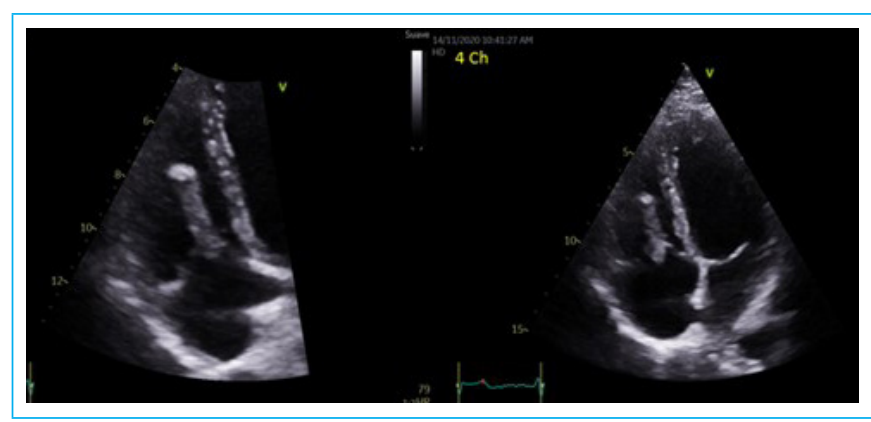

Figura 1. Masa ecodensa móvil en el ventrículo derecho altamente sugestiva de trombo que en ocasiones se desplaza hasta el nivel de la válvula tricúspide (31×8 mm, área $\left.2.4 \mathrm{~cm}^{2}\right)$.

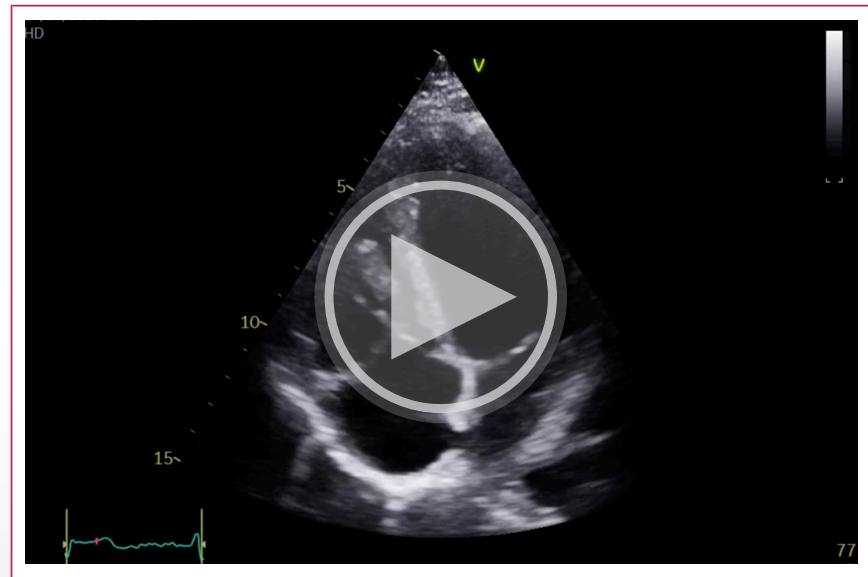

Vídeo 1. Trombo en tránsito en el VD: Vista 4C.

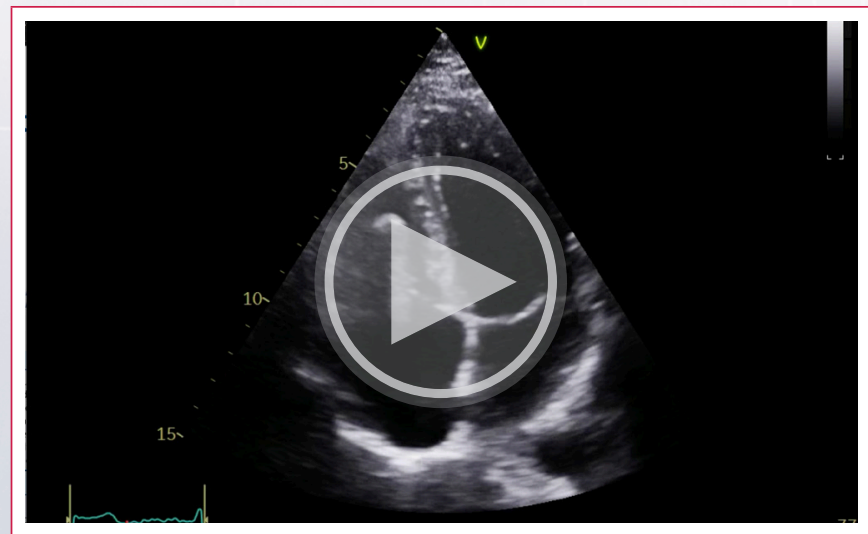

Vídeo 2. Trombo en tránsito en el VD: Zoom vista 4Ch.

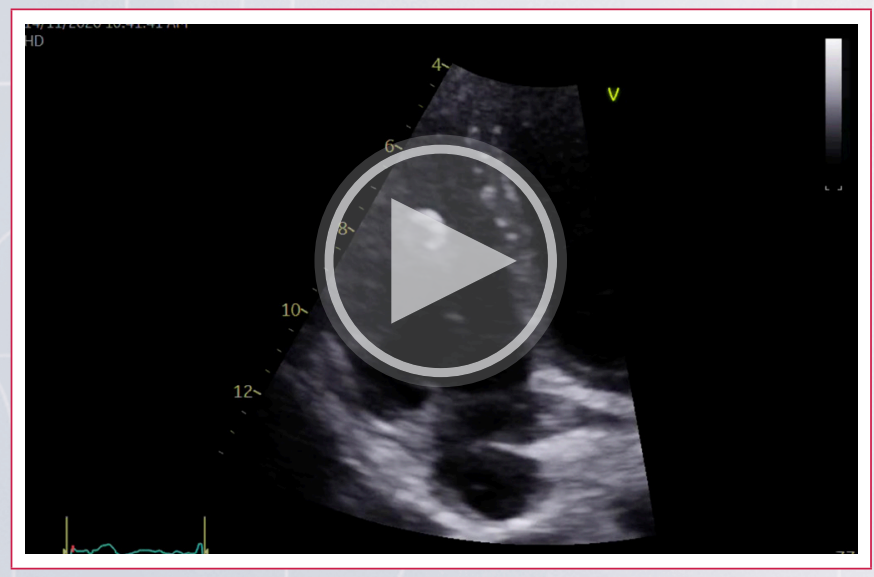

Vídeo 3. Trombo en tránsito en el VD: Zoom vista 4Ch.

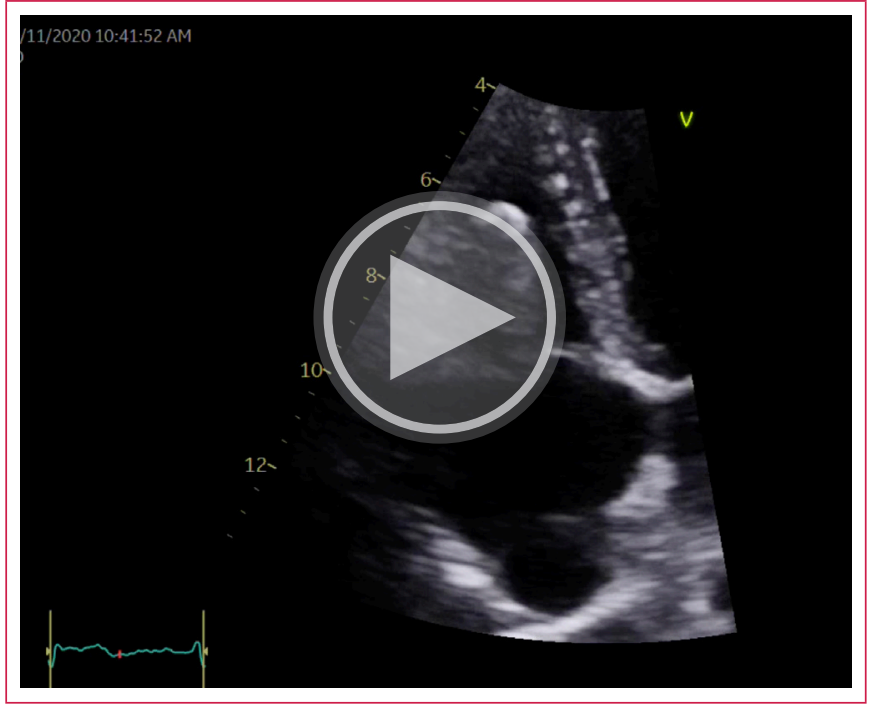

Vídeo 4. Trombo en tránsito en el VD: Zoom vista 4Ch.

Al ingreso a urgencias, estable hemodinámicamente, sin alteraciones a nivel cardiopulmonar, electrocardiograma dentro de parámetros normales, se inició anticoagulación con heparina de bajo peso molecular ajustada al peso, se ampliaron estudios donde se descartó enfermedad tromboembólica venosa. Ante la sospecha de trombo en tránsito se realizó resonancia cardíaca, la cual confirmó la presencia de lesión en la porción trabecular del VD sin captación del contraste en la perfusión dinámica ni en las secuencias de realce tardío. No se demostraron otras lesiones en el ventrículo, válvulas, aurícula derecha ni en las arterias pulmonares. La morfología y función biventricular estaban normales: FEVI 67\%-TAPSE 23 $\mathrm{mm}$, sin signos de fibrosis endomiocárdica ni otros hallazgos que sugirieran enfermedad infiltrativa (ver figura 2, ver videos 5-8). Los hallazgos descritos en la resonancia eran característicos de trombo móvil en tránsito en el VD, por tal motivo para definir la etiología de éste fue valorada por hematología quienes consideraron estudios basales, tamización para síndrome antifosfolípido (SAF), pruebas moleculares para trombofilia, perfil infeccioso.

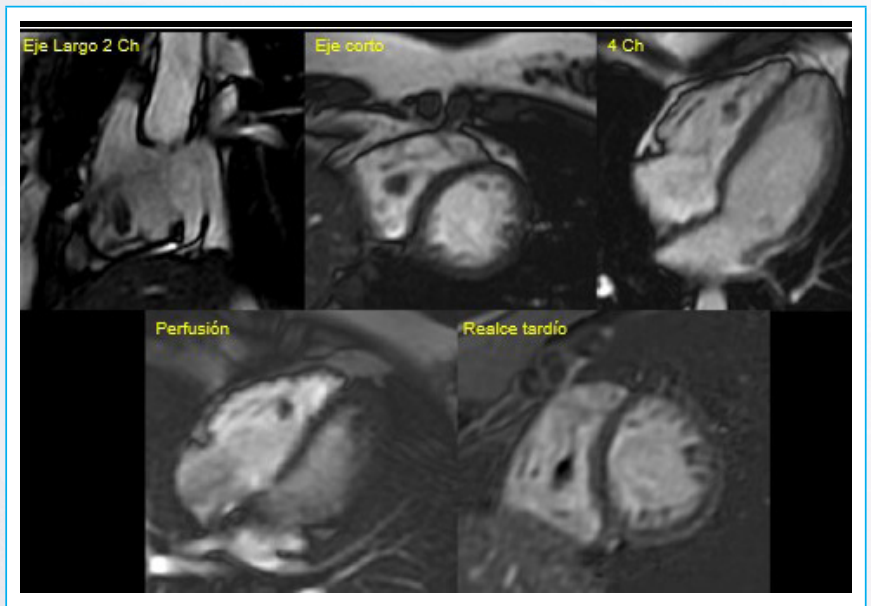

Figura 2. Las imágenes cine en los diferentes planos cardiacos demuestran una lesión hipo intensa en la porción trabecular del ventrículo derecho, con un diámetro máximo de $2 \mathrm{~cm}$, la cual es móvil. Las imágenes dinámicas de perfusión en reposo demuestran defecto de llenado y las secuencias de realce tardío con gadolinio no evidencian captación en la lesión. Por los hallazgos es característico de trombo móvil y no se encuentran hallazgos que sugieran neoplasia. 


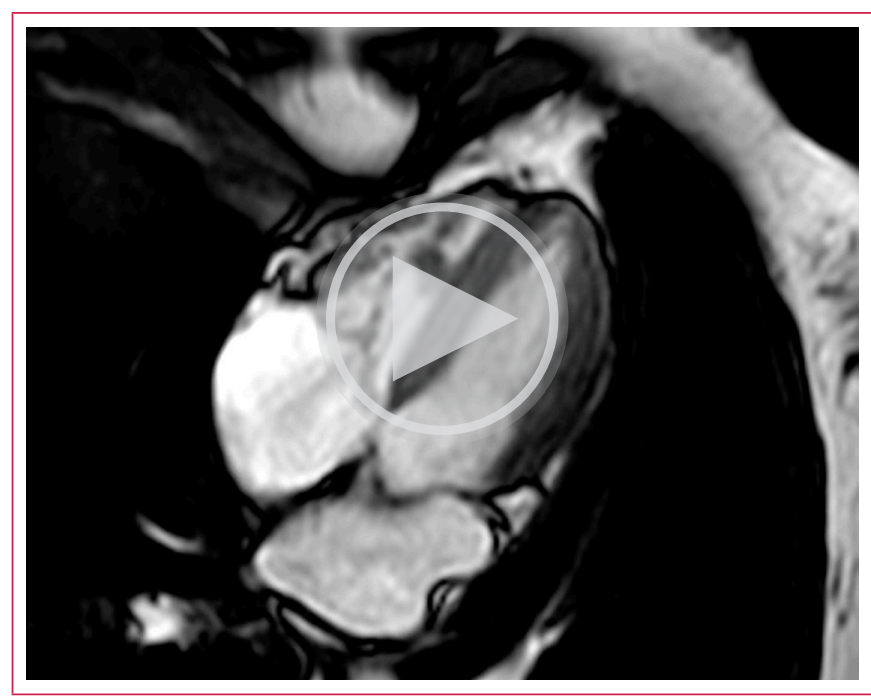

Vídeo 5. Trombo en el VD: Vista 4Ch.

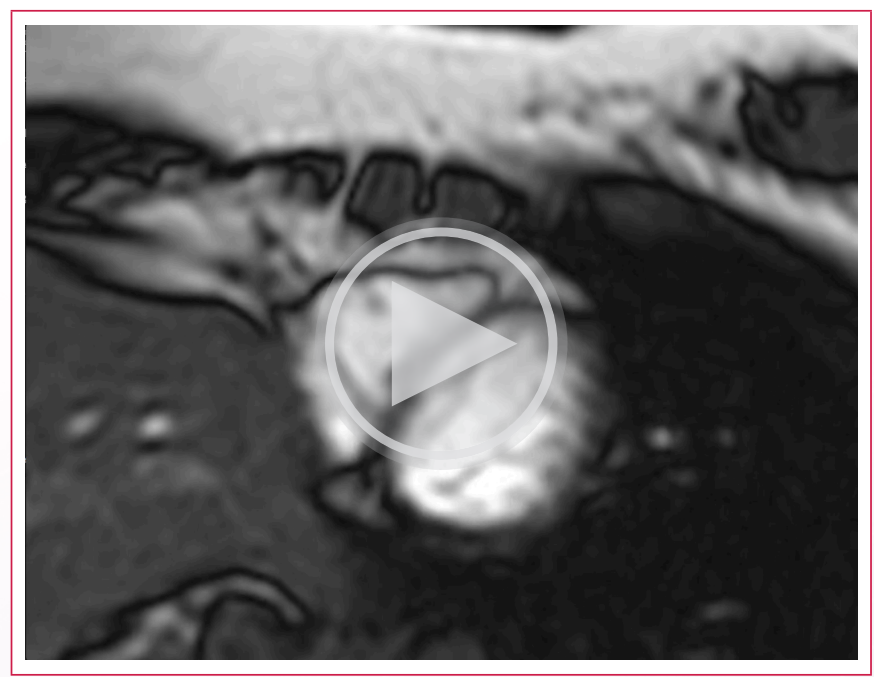

Vídeo 6. Trombo en el VD: Eje corto.

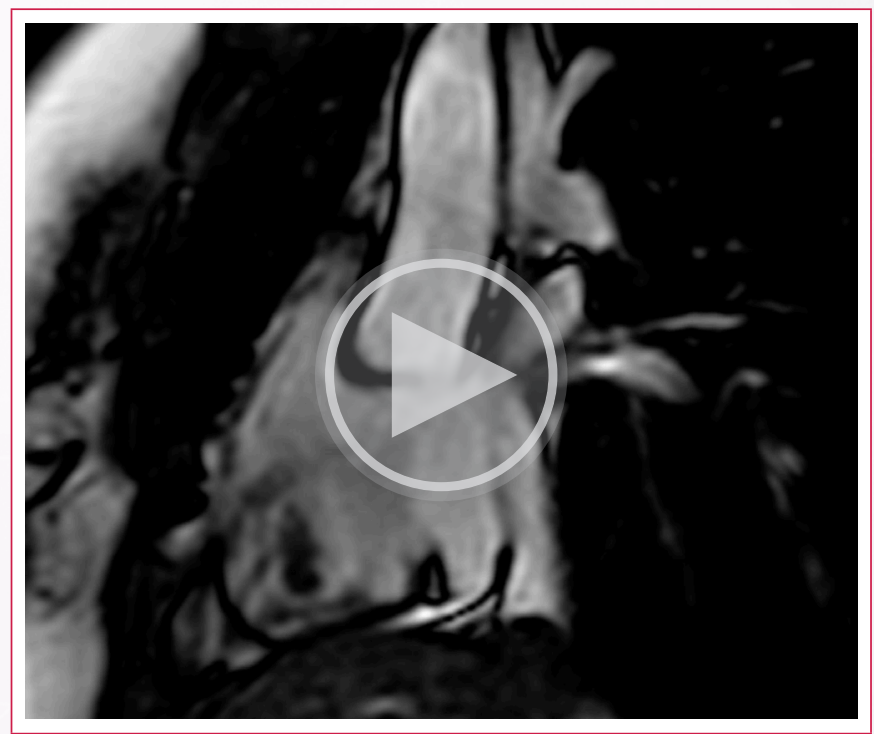

Vídeo 7. Trombo en el VD: Eje largo del VD.

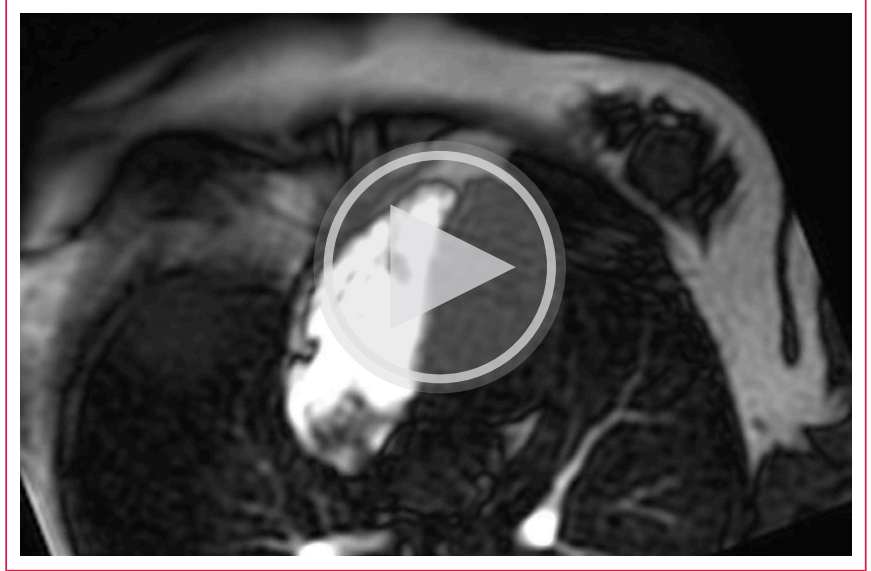

Vídeo 8. Trombo en el VD: Perfusión.

Por cardiología se consideró un trombo en tránsito en el VD, el cuál le confería un alto riesgo de embolia pulmonar y se indicó resección quirúrgica. Cirugía cardiovascular la lleva a cirugía, obteniendo una masa que macroscópicamente sugería fibroelastoma de $1 \mathrm{~cm}$ de longitud originado en cuerda tendinosa del aparato subvalvular de la válvula tricúspide con trombo adherido de $3 \mathrm{~cm}$ de longitud muy móvil. Ingresa a la unidad de cuidado intensivo para vigilancia con una evolución satisfactoria, logrando retiro de ventilación mecánica, drenes de pericardio y tórax. Hematología revisó los estudios descritos previamente, los cuales fueron negativos y debido a los hallazgos macroscópicos en cirugía deciden continuar anticoagulación de manera indefinida y ordenan estudios ambulatorios.

Posteriormente patología informa que se descarta fibroelastoma y que el hallazgo histopatológico es un trombo antiguo en la base más trombo de origen reciente en el resto de la masa. El ETT de control mostró una insuficiencia tricuspídea grado II/IV, el cual no requería intervenciones adicionales.

En controles ambulatorios se evidenció en repetidos exámenes anticoagulante lúpico positivo por lo que se determina una alta probabilidad de síndrome antifosfolípido.

\section{Discusión}

Las masas evidenciadas en el VD requieren un estudio imagenológico multimodal para orientar una terapéutica ideal. Este caso lo ilustra. En la evaluación no invasiva de masas cardíacas se incluye la ecocardiografía, la tomografía y la resonancia magnética. La ecocardiografía puede mostrar la ubicación anatómica, la extensión, las consecuencias fisiológicas durante el ciclo cardíaco. Si la masa tiene localización posterior se observa mejor por la ecocardiografía transesofágica.

Las imágenes ecodensas sugieren trombo si la ecocardiografía de contraste muestra que la masa no tiene tallo (los trombos casi nunca tienen tallo), la cámara auricular está agrandada, el gasto cardíaco es bajo, hay estasis y la masa es avascular. Si hay anormalidades de la contractilidad cerca a la masa, favorece que sea trombosis en vez de tumor', sin embargo, en Colombia actualmente no se cuenta con contraste ecocardiográfico para discriminar entre tumor o trombo.

Las vegetaciones son generalmente de apariencia irregular, móviles y unidas a una válvula, las grandes se asocian más a compromiso por hongos. El lado izquierdo del corazón suele comprometerse más que el derecho'. 
Tanto la tomografía como la resonancia cardíaca permiten precisar la localización anatómica, la caracterización tisular y el manejo perioperatorio de las masas cardíacas grandes. La ventaja de la resonancia cardíaca es que ofrece un campo de visión más amplio, contraste de tejido superior, versatilidad en planos de imagen y capacidad única en la caracterización tisular. Por lo anterior, se considera el examen de elección para la caracterización de masas cardíacas, frecuentemente evidenciadas en otros estudios imagenológicos ${ }^{2,3}$.

En el caso de la paciente, se descartó enfermedad tromboembólica venosa y la resonancia cardíaca permitió caracterizar la lesión definiéndola como trombo en VD, este hallazgo observado en pacientes aparentemente estables es de peor pronóstico ${ }^{4}$, además, la resonancia magnética cardíaca permitió descartar otras posibles etiologías como neoplasias, enfermedades infiltrativas, cardiopatía arritmogénica del VD, entre otras ${ }^{2.5}$. Teniendo la certeza imagenológica de un trombo en el VD se llevó a resección por cirugía cardíaca. Cabe resaltar, existen diferentes etiologías de un trombo en VD, desde trombofilias, neoplasias activas, infección por SARS-CoV- $2^{6}$ y causas poco comunes como un síndrome nefrótico ${ }^{7}$.

La multimodalidad en la imagen orienta al clínico sobre la posible etiología de una masa en el VD, se han descrito imágenes híbridas como la tomografía con emisión de positrones con 18F-Fluorodesoxiglucosa y resonancia magnética cardíaca (18F-FDG-PET/CMR, por sus siglas en inglés)', pero la histopatología será finalmente la que confirma el diagnóstico

\section{Conclusiones}

Los trombos en tránsito en el VD son poco frecuentes, generalmente se encuentra asociados a enfermedad tromboembólica venosa. La imagen multimodal es vital, porque determina la localización anatómica, caracterización, manejo perioperatorio y el seguimiento que se debe realizar.

\section{Ideas para recordar}

- La imagen multimodal es fundamental en la evaluación de masas intracardíacas.

- La diferenciación de trombo, tumor o vegetación por medio de la imagen multimodal, orienta al clínico en el diagnóstico y tratamiento.

\section{Bibliografía}

1. Patnaik S, Shah M, Sharma S, Ram P, Rammohan HS, Rubin A. A large mass in the right ventricle: Tumor or thrombus? CCJM. 2017;84(7):517-9.

2. Maini R, Gadiraju TV, Jabbar A, Danrad R, Sweeney A. Cardiac MRI as a useful tool to differentiate tumor and thrombus. Int J Cardiovasc Imaging. 2017;33(11):1795-6

3. Cely CA, López-Guarch CJ, Charterina SA. Masas cardiacas: multimodalidadresonancia magnética cardiaca. Revista Colombiana de Cardiología. 1 de julio de 2019;26:111-22

4. Rai MP, Herzallah K, Alratroot A, Laird-Fick H. The dilemma in the management of haemodynamically stable pulmonary embolism with right heart thrombus. BMJ Case Reports CP. 2019;12(7):e229162.

5. Sušić L, Baraban V, Vincelj J, Maričić L, Ćatić J, Blažeković R, et al. Dilem$m a$ in clinical diagnosis of right ventricular masses. J Clin Ultrasound. 2017;45(6):362-9.

6. Sethi Sanjum S., Zilinyi Robert, Green Philip, Eisenberger Andrew, Brodie Daniel, Agerstrand Cara, et al. Right ventricular clot in transit in covid-19. JACC: Case Reports. 2020;2(9):1391-6.

7. Lempp S, Schwenger V. Isolated right ventricular thrombus in an adult patient with nephrotic syndrome: a case report. J Med Case Rep. 2017;11(1):311.

8. Benz DC, Fuchs TA, Tanner FC, Eriksson U, Yakupoglu HY. Multimodality imaging of a right ventricular mass. Eur Heart J Cardiovasc Imaging. 2019;20(10):1184. 